\title{
FINE SPLITTING IN A POTENTIAL MODEL INCORPORATING RELATIVISTIC KINEMATICS
}

\author{
I. Haysak, V. Lengyel, A. Shpenik \\ Uzhgorod State University, Chair of Theoretical Physics, 32 Volosin Str., Uzhgorod UA-294000, Ukraine
}

(Received January 22, 1996)

\begin{abstract}
The problem of two quarks interaction is reducet to the solution of one particle Dirac equation. It was solved analytically and numerically using the potential like $V=-\alpha / r+A r$. We obtained the spin-orbital mass differences in different mesons.
\end{abstract}

Key words: quarks, potential model, oscillator, Dirac equation.

PACS number(s): 12.40; $12.90 ; 14.40$

The description of hydrogen-like mesons as bound states of quarks of different masses by potential models arouse considerable interest. Even quite recently extensive relativistic (semirelativistic) or nonrelativistic calculations were carried out (see [1-4] and the references there in). Both types of models use a certain kind of "QCD-motivated" two-body interaction potential. Most frequently this is a potential consisting of the one-gluon exchange term $V_{g}(r)$ and confining the long range part $V_{c}(r)$. So the basic Anzats lies in expressing the interaction in the form of

$$
V(r)=V_{g}+V_{c}+V_{0}
$$

where $V_{g}=-\alpha / r$ is the one gluon exchange, $V_{c}=A r$ is the long range confinement part and $V_{0}$ fixes the ground state.

Most of the researchers use the nonrelativistic or semirelativistic potential models. Such an approach gives a more or less good description of radial and orbital excitations of mesons but the spin-orbital effects calculated within the perturbation method can not be anything but unreliable.

The numerical solutions in relativistic as well as in nonrelativistic approaches yield a farly good description of radial and orbital excitations of mesons. Spin-orbital effect in quasirelativistic as well in nonrelativistic models are obtained within the framework of the so called Generalized Breit-Fermi approach [1] where it has the form

$$
V_{S L}(r)=\frac{1}{2 m^{2} r} \frac{d V}{d r} \mathbf{S} \cdot \mathbf{L} \text {. }
$$

For Coulomb-like potential in semirelativistic Shroedinger equation it gives the $1 / r^{3}$ term which leads to the problem of a particle falling on the force center and therefore the fine structure of mesons is calculated usually only as a perturbation for the central part of the potential.

The main purpose of the present work is to estimate spin-orbital splitting of meson spectra in the frame of the Dirac equation which should hopefully describe these phenomena more acurately. Such a model could be real- istic for the hydrogen-like system of bounded light and heavy quarks.

The application of a one-particle Dirac equation to the two-quark system has been thoroughly discussed [5, 6 , 7]. In order to observe why in the Dirac approach we do not encounter the problem of the particle falling to the force center as in the semirelativistic one let us consider the Dirac equation for the radial functions $f(r)$ and $g(r)$ of the Dirac spinor

$$
(E-v(r)-s(r)-m) f(r)=-\frac{k}{r} g(r)-\frac{d g(r)}{d(r)}
$$

$$
(E-v(r)+s(r)+m) g(r)=-\frac{k}{r} f(r)+\frac{d f(r)}{d r},
$$

where

$$
k=\left\{\begin{array}{cc}
l+1, & \text { for } j=l+1 / 2, \\
-l, & \text { for } j=l-1 / 2,
\end{array}\right\}
$$

$j$ is the total angular momentum; $l$ is the orbital angular momentum; $E$ is the total energy of one particle; $m$ is the quark mass; $v(r)$ is the vector part of the potential; $s(r)$ is the scalar part of the potential; $f(r)$ and $g(r)$ are the radial wave-functions.

For our purpose of finding the energy $E$ it is sufficient to consider, as usual, only the solution of the radial equation for a large component $f(r)$. By eliminating $g(r)$ from (3) and introducing a new function $\varphi(r)$ according to

$$
f(r)=\sqrt{s(r)-v(r)+m+E} \varphi
$$

we obtain a single second order differential equation for $\varphi(r)$ :

$$
\varphi^{\prime \prime}+\left[\frac{(s(r)-v(r))^{\prime \prime}}{2 D}-\frac{3(s(r)-v(r))^{\prime 2}}{4 D^{2}}\right.
$$




$$
\begin{aligned}
& +\frac{(s(r)-v(r))^{\prime \prime} k}{D r}-\frac{k(k-1)}{r^{2}} \\
& +(E-v(r)+s(r)+m)(E-v(r)-s(r)-m)] \varphi=0
\end{aligned}
$$

where

$$
D=E-v(r)+s(r)+m .
$$

Let us now consider the third term in square brackets (5) which is responsible for the fine-splitting. Note that $D^{\prime}=(s(r)-v(r))^{\prime} \rightarrow v(r)^{\prime}$ at $r \rightarrow 0$ in the case that $v(r)$ is the Colummb-like potential $\left(v(r)=V_{g}\right)$. Then

$$
\begin{aligned}
& \frac{D^{\prime}}{D r} \rightarrow \frac{1}{2 m r} \frac{d V_{g}}{d r}, \quad m \gg E \gg V_{g}, \\
& \frac{D^{\prime}}{D r} \rightarrow \frac{1}{r V_{g}} \frac{d V_{g}}{d r}, \quad V_{g} \gg m, \quad V_{g} \gg E, \quad(r \rightarrow 0) .
\end{aligned}
$$

The nonrelativistic limit for the fine-spitting term is usually obtained by putting $E+m \simeq 2 m \gg\left|V_{g}\right|$ and from (6) one obtains $\frac{1}{2 m r} \frac{d V_{g}}{d r}$ i.e. the term of type (2). But if $r \rightarrow 0$ one cannot put $E+m \succ V_{g}$ and just the opposite limit is to be taken, namely $E+m \ll V_{g}$ at $r \rightarrow 0$. So in the consequent relativistic approach one obtains the expression $\frac{1}{r V_{g}} \frac{d V_{G}}{d r}$ for spin-orbital splitting. The result is $1 / r^{3}$-type behaviour in the nonrelativistic limit for Coulomb-like potential, while $1 / r^{2}$ is obtained in the relativistic limit. So the problem of falling to the center is eliminated in the relativistic approach.

The equation (3) can be solved numerically as well as analytically. Let us begin from the description of the analytical solution.

While dealing with the Dirac equation one encounters the problem of Lorentz structure of the potential (see e. g. [1]). Many are inclined to believe that the only gluon exchange is of the character of the vector, the confinement being a scalar. Our numerical as well analytical calculations show that it does not seem to be so. We are convinced that in order to obtain the correct sign of spin orbital splitting in Dirac equation it is necessary to take the confinement part of the potential partly for the vector character since in the opposite case one obtains $M\left(P_{3 / 2}\right)<M\left(P_{1 / 2}\right)$. It is important to point out that a similar conclusion concerning the Lorentz character of the confinement was made by $\mathrm{H}$. Crater, P. Van Alstine [6]. We study the following Lorentz structure of the potential

$$
v(r)=V_{g}+\varepsilon V_{c}, \quad s(r)=(1-\varepsilon) V_{c},
$$

where $\varepsilon$ is the parameter which indicates the part of confining interaction in the vector sector of the potential. It turns out that the larger part of the confinement $(\varepsilon=0.48 \div 0.65)$ must be of a vector character. Now we understand why Moshinsky et al [8] obtain too large contribution in the spin-orbital interaction with Dirac equation. It happened because of their suggestion of a totally scalar character of the confinement. In the physically interesting region $(0 \div 1 \mathrm{fm}) D \rightarrow \frac{\alpha}{r}$. After applying this limit in (5) we obtain

$$
\varphi^{\prime \prime}+\left[E^{2}-m^{2}-2 A \alpha \varepsilon+(1-2 \varepsilon) \frac{A k}{\alpha}+(1-2 \varepsilon) \frac{3 A}{2 \alpha}-\left((1-2 \varepsilon)^{2} \frac{3 A^{2}}{4 \alpha^{2}}+(1-2 \varepsilon) A^{2}\right) r^{2}+\frac{\alpha^{2}-k^{2}+1 / 4}{r^{2}}\right] \varphi=0
$$

In this equation we neglect the term $(2 A m r-2 E \alpha / r)$ for its being numerically small. It is easy to see that equation (8) is an oscillator equation. The solution of this simplified equation exists at energy eigenvalues

$$
E^{2}=\left(4 N+2+2 \sqrt{k^{2}-\alpha^{2}}\right) \sqrt{(1-2 \varepsilon)^{2} \frac{3 A^{2}}{4 \alpha^{2}}+(1-2 \varepsilon) A^{2}}+m^{2}+2 \varepsilon \alpha A-(1-2 \varepsilon) \frac{A k}{\alpha}-(1-2 \varepsilon) \frac{3 A}{\alpha} .
$$

From this expression we can see that in order to obtain a correct sign of the spin-orbital splitting it is necessary to take $\varepsilon$ to be larger than $0.48 \div 0.65$. That indicates that the confinement must rather be a vector. Expression (9) is the solution of Dirac equation (3) with potential (1).
We used the analytical solution to show the direction in which we must search the parameters for the numerical solution. We calculated, for example, the fine splitting according to expression (9) in $s \bar{u}$ system with the following parameters: $m_{s}=0.27 \mathrm{GeV}, m_{u}=0.005 \mathrm{GeV}, A=0.18$ 
$\mathrm{GeV}^{2}, \varepsilon=0.48$. The result is $\Delta\left(P_{3 / 2}-P_{1 / 2}\right)=72 \mathrm{MeV}$ while the experiment gives $68 \mathrm{MeV}$. These parameters are chosen by fixing the basic mass spectrum [11].

In obtaining the values of meson masses we proceed from the argumentation given in the work of Predazzi et al [9]. In this paper the authors start from the classical relativistic relation for two body systems $W=$ $\sqrt{p_{1}^{2}+m_{1}^{2}}+\sqrt{p_{2}^{2}+m_{2}^{2}}$. In the system of the center of mass $\mathbf{p}_{1}=\mathbf{p}_{2}$ and with an elementary transformation this leads to the one particle Dirac equation $c \mathbf{p} \boldsymbol{\alpha}+\beta m_{1} c^{2}=E$ where $W=E+\sqrt{E^{2}-m_{1}^{2}+m_{2}^{2}}$ is the total energy of a two particle system (i.e. the mass of the bound state system, $M=W$ is the meson mass), $W_{1}$ being the total energy of one particle.

Now let us solve equation (3) numerically. The radial functions $f(r)$ and $g(r)$ in equation (3) obey the boundary conditions $f(0)=0, g(0)=0, f(\infty)=0, g(\infty)=0$.

The first two boundary conditions are used as the starting conditions and the second pair of boundary conditions can be used for the selection of eigenvalues of energy in equation (3) under the numerical solution by Runge-Kutta method.

\begin{tabular}{|c|c|c|c|c|c|c|c|c|c|c|}
\hline & \multicolumn{2}{|c|}{$b \bar{b}$} & \multicolumn{2}{c|}{$c \bar{c}$} & \multicolumn{2}{c|}{$b \bar{s}$} & \multicolumn{2}{c|}{$c \bar{u}$} & \multicolumn{2}{c|}{$s \bar{u}$} \\
\hline & $M_{t h}$ & $M_{e x p}$ & $M_{t h}$ & $M_{e x p}$ & $M_{t h}$ & $M_{e x p}$ & $M_{t h}$ & $M_{e x p}$ & $M_{t h}$ & $M_{e x p}$ \\
\hline $1 \mathrm{~S}$ & 9460 & 9460 & 3106 & 3096 & 2094 & 2110 & 2016 & 2010 & 884 & 892 \\
\hline $2 \mathrm{~S}$ & 10016 & 10023 & 3596 & 3686 & 2554 & & 2448 & & 1316 & 1410 \\
\hline $3 \mathrm{~S}$ & 10344 & 10355 & 3942 & 4040 & & & 2787 & & & \\
\hline $4 \mathrm{~S}$ & 10603 & 10580 & & & & & & & & \\
\hline $5 \mathrm{~S}$ & 10826 & 10865 & & & & & & & & \\
\hline
\end{tabular}

Table 1. Radial excitation of $(q \bar{q})$-system (masses are given in $\mathrm{MeV}$ )

\begin{tabular}{|lllll|}
\hline & $M_{e x p}$ & $M_{t h}[2]$ & $M_{t h}$ & \\
\hline${ }^{1} P_{1}$ & 1270 & 1350 & & \\
& & & 1113 & $P_{1 / 2}$ \\
${ }^{3} P_{0}$ & 1350 & 1240 & & \\
& $1430 ?$ & & & \\
\hline${ }^{3} P_{1}$ & 1406 & 1370 & & \\
& & & 1237 & $P_{3 / 2}$ \\
${ }^{3} P_{2}$ & 1430 & 1430 & & \\
${ }^{1} D_{2}$ & - & & 1412 & $D_{3 / 2}$ \\
& & & & \\
${ }^{3} D_{1}$ & 1670 & & & \\
${ }^{3} D_{2}$ & 1770 & & 1484 & $D_{5 / 2}$ \\
& & & & \\
\hline${ }^{3} D_{3}$ & 1780 & & & \\
\hline
\end{tabular}

Table 2. $(s \bar{u})$-system (masses are given in MeV)

\begin{tabular}{|c|c|c|c|}
\hline \multicolumn{4}{|c|}{$M_{e x p} \quad M_{t h}$} \\
\hline \multicolumn{4}{|c|}{$c \bar{u}$} \\
\hline $\begin{array}{l}{ }^{1} P \\
{ }^{3} P\end{array}$ & $\begin{array}{l}- \\
-\end{array}$ & 2232 & $P_{1 / 2}$ \\
\hline $\begin{array}{l}{ }^{3} P \\
{ }^{3} P\end{array}$ & $\begin{array}{l}2420 \\
2460\end{array}$ & 2355 & $P_{3 / 2}$ \\
\hline \multicolumn{4}{|c|}{$c \bar{s}$} \\
\hline${ }^{1} P$ & $\begin{array}{r}2536 \\
-\end{array}$ & 2477 & $P_{1 / 2}$ \\
\hline $\begin{array}{l}{ }^{3} P \\
{ }^{3} P\end{array}$ & $\begin{array}{l}- \\
-\end{array}$ & 2577 & $P_{3 / 2}$ \\
\hline
\end{tabular}

Table 3. $(c \bar{u}, c \bar{s})$-systems (masses are given in $\mathrm{MeV}$ )

The following parameters are used in the numerical calculations $\alpha=0.5, A=0.18 \mathrm{GeV}^{2}$ which correspond to the usually accepted value for Cornell potential and $\varepsilon=0.65$.

As for the case of equal quark masses the Dirac equation gives the spin-orbital splitting only into two levels while the experiment gives three levels. It is due to the fact that the individual quark spins can add to 0 or 1 and the total angular momentum can be 0,1 or 2 in the cases of $l=1$. But in the limit of an infinitely heavy quark mass the heavy and light degrees of freedom decompose and the light degrees of freedom determine the quantum states, the total angular momentum of the light degrees of freedom being $j=L+s$. This gives two sets of levels $j=1 / 2, j=3 / 2$. For example, in the hydrogen atom, we do not worry about the nuclear spin - it enters only as a hyperfine effect [10]. In comparing our data with other data in terms of total momentum we kept to the following rule: $P_{1 / 2}$ was considered as being an averaged mixture of ${ }^{1} P_{1}$ and ${ }^{3} P_{0}$, while $P_{3 / 2}$ was considered as averaged mixture of ${ }^{3} P_{1}$ and ${ }^{3} P_{2}$ masses. This problem can have a different solution and it will be discussed elsewhere.

The numerical results are shown in tables 1, 2, 3. As we can see, the mass spectrum (Table 1) is obtained with reasonable accuracy [11]. As for fine splitting for $s \bar{u}$-system (Table 2) we have splitting $\Delta=124 \mathrm{MeV}$ (108 MeV exp.) and $\Delta=72 \mathrm{MeV}$ (95 MeV exp.) for $P$ and $D$-waves correspondingly. Other data (Table 3 ) can be considered as predictions. The assessuent of Godfrey, Kokoski's splitting for the $s \bar{u}$ system for $P$-wave according to our prescription and also that given in [10] yield $105 \mathrm{MeV}$. Unfortunatly Godfrey did not calculate the $D$ wave splitting.

The problem of two-quark systems with different masses was also examined in [2] and an excellent overview of the situation is given in [12]. Comparing our results with those obtained in [2] we shall see that the 
splitting in $s \bar{u}$-system is quite similar, however the splitting in $c \bar{u}$-system obtained in [2] is much smaller. The absence of experimental data does not allow us to make the final conclusion. It is interesting to note that both ours and Godfrey and Kokoski's [2] results strongly suggest that the value of ${ }^{3} P_{0} s \bar{u}$-mass equaling $1430 \mathrm{MeV}$ is too large and the previous one of $1350 \mathrm{MeV}$ [13] is preferable. It is also important to underline that:

1. It is promising to carry out successful calculations by Dirac equation in hydrogen-like systems both for the mass spectrum and fine splitting;

2. Introducing the Lorentz character of the confinement as admixture of scalar and vector types we estab- lished them to be roughly equal which is confirmed by both numerical and analytical methods of calculations of Dirac equation. In conclusion we would like to point out that very interesting quark masses sum rules follow from eq. (9). This question was discussed in [14].

\section{ACKNOWLEDGEMENT}

Out research was partly supported by the International Soros Science Education Program of the International Renaissance Foundation, GRANT SPU 042037. We also thank O. Zatsarinny for his valuable remarks.
[1] W. Lucha, F. Schoberl, Phys. Rep. 200, 127 (1991).

[2] S. Godfrey, R. Kokoski, Phys. Rev. D 43, 1679 (1991).

[3] C. Semay, B. Silvestre-Brac, Phys. Rev. D 46, 5177 (1992).

[4] S. Chalupka, V. Lengyel, M. Salak, Czech. J. Phys. 44 107 (1991).

[5] S. Deoghuria, S. Chakrabarty, J. Phys. G 15, 1213 (1989).

[6] H. Crater, Van Alstine, Phys. Rev. D 37, 1982 (1988).

[7] C. Semay, R. Ceuleneer, Phys. Rev. D 48, 4361 (1993).

[8] V. I. Kukulin, G. Loyola, M. Moshinsky, Phys. Let. A 158, 19 (1991).
[9] D. B. Lichtenberg, E. Predazzi, C. Rossetti, Z. Phys. C, Particles and Fields 40, 357 (1988).

[10] J. N. Butler, S. Shukla, Preprint Fermilab - Conf - 95/049 (1995) p. 15.

[11] Rev. of Part. Prop., Phys. Rev. D 45, (1992).

[12] N. Isgur, XXVI Int. Conf. on High En. Phys., Dallas 1992 CEBAF-TH-92-31.

[13] Rev. of Part. Prop., Phys. Lett. B 170, (1986).

[14] I. Haysak, V. Lengyel, A. Shpenik, 15 European FewBody conference, Peniscola, Spain, (1995) book of abstract, p. 36.

\title{
ТОНКЕ РОЗЩЕПЛЕННЯ В ПОТЕНЦАЛІ З РЕЛЯТИВІСТИЧНОЮ КІНЕМАТИКОЮ
}

\author{
I. Гайсак, В. Лендьел, О. Шпеник \\ Уэгородсъкий держсавний університет, кафедра теоретичної фізики \\ Україна, UA-294000, Ужгород, вул. Волошина, 32
}

\footnotetext{
Проблема взаємодії двох кварків зведена до розв'язання одночастинкового рівняння Дірака. Останнє розв'язується як наближено аналітично, так і чисельно з використанням потенціалу типу “лійки" $V=$ $-\alpha / r+A r$. Особливістю використаного підходу є можливість точного врахування спін-орбітальної взаємодії без зведення до наближених методів на кшталт метода збурень. Розраховано спін-орбітальну різницю мас мезонів, які складаються як з одинакових кварків, так і з кварків з різними масами. Порівняння з експериментом вказує на добру узгодженість теорії з експериментом.
} 